\title{
Survival and long-term toxicities of pediatric Hodgkin lymphoma after combined modality treatment: a single institute experience
}

\author{
Kye Jin Song ${ }^{1}$, Jin-hong Park ${ }^{1}$, Ho Joon Im² $^{2}$ Seung Do Ahn ${ }^{1}$ \\ ${ }^{1}$ Department of Radiation Oncology, Asan Medical Center, University of Ulsan College of Medicine, Seoul, Korea \\ ${ }^{2}$ Department of Pediatrics, Asan Medical Center Children's Hospital, University of Ulsan College of Medicine, Seoul, Korea
}

Received: June 5, 2020

Revised: August 11, 2020

Accepted: August 17, 2020

Correspondence:

Seung Do Ahn

Department of Radiation Oncology,

Asan Medical Center, University of

Ulsan College of Medicine, 88

Olympic-ro 43-gil, Songpa-gu, Seoul

05505, Korea.

Tel: $+82-2-3010-4436$

Fax: +82-2-3010-6950

E-mail: sdahn@amc.seoul.kr

ORCID:

https://orcid.org/0000-0003-4153-4191
Purpose: To analyze the clinical outcomes and long-term toxicity of pediatric patients with Hodgkin lymphoma after combined-modality treatment (CMT) with involved-field or involved-nodal radiotherapy (RT).

Materials and Methods: We retrospectively reviewed the records of 27 pediatric Hodgkin lymphoma patients who received CMT at a single institution between January 1990 and July 2017. Patients with stage I-III received a heterogeneous chemotherapy regimen depending on their risk group followed by 19.8-36 Gy RT, with the dose based on their response to the chemotherapy before RT. An optional 9-20 Gy boost was delivered to residual sites. The risk group was determined based on the initial stage, the presence of bulky disease, and any B symptoms. We evaluated overall survival, event-free survival, and long-term toxicities.

Results: A total of 27 patients completed the CMT. At a median follow-up of 125 months (range, 9 to 337 months), the estimated 5-year event-free survival and overall survival were $88.9 \%$ and $96.3 \%$, respectively. Late symptomatic cardiopulmonary toxicity was not observed, and only one patient was positive on a subclinical obstructive pulmonary function test. The incidence of hypothyroidism was 58.3\% among 12 patients with an available thyroid function test. There was one papillary thyroid cancer diagnosed 7.2 years after treatment.

Conclusion: CMT for pediatric Hodgkin lymphoma with involved-field and involved-nodal RT achieved an excellent survival with only modest long-term toxicity. Smaller-field RT seemed to decrease long-term toxicities and had good local control.

Keywords: Pediatrics, Hodgkin disease, Radiotherapy, Survival, Long-term adverse effects

\section{Introduction}

Pediatric Hodgkin lymphoma $(\mathrm{pHL})$ is a rare disease in Korea with a crude incidence of 1.3 per million among ages 0 to 14 years [1]. In the United States, pHL accounts for nearly 5\%-10\% of pediatric malignancies, with approximately 1,700 newly diagnosed cases among children under 20 years old [2]. Studies conducted in western countries report that $\mathrm{pHL}$ is a highly curable disease with 5-year event-free survival (EFS) and overall survival (OS) exceeding
90\% [3]. However, the epidemiology and clinicopathologic features of Hodgkin lymphoma in Korea show discrepancies with the features of western countries [4].

High-dose mantle-field radiotherapy (RT) ensures long-term survival of greater than $80 \%$, but long-surviving patients experience unacceptable complications, including cardiovascular and endocrinal morbidity and secondary malignancies [5]. Therefore, recent studies have applied risk-adapted chemotherapy combined with low-dose RT, and they demonstrated long-term survival and EFS

Copyright (C) 2020 The Korean Society for Radiation Oncology

This is an Open Access article distributed under the terms of the Creative Commons Attribution Non-Commercial License (http://creativecommons.org/licenses/by-nc/4.0/) which permits unrestricted non-commercial use, distribution, and reproduction in any medium, provided the original work is properly cited. 
over 90\% [6-10]. Contemporary radiation techniques with involved-field radiotherapy (IFRT) or involved-nodal radiotherapy (INRT) have lower risks of long-term toxicities.

There are only a few studies that have reported on Hodgkin lymphoma, but most of them have included the entire age group due to the extremely low incidence of $\mathrm{pHL}$ in Korea. Only one study specifically reported the clinical features and survival of pediatric malignant lymphoma cases, including 18 patients with $\mathrm{pHL}$, but no clinical data about the treatment modality or RT techniques were described [11]. Moreover, there is no report on the survival or treatment-related toxicities of $\mathrm{pHL}$ among pediatric and adolescent cases in Korea that received contemporary combined-modality treatment (CMT). Therefore, we observed the clinical outcomes and long-term toxicity of pediatric patients with Hodgkin lymphoma after CMT with IFRT or INRT in a single institute.

\section{Materials and Methods}

We reviewed the medical records of pediatric patients under 19 years old with Hodgkin lymphoma between January 1990 and July 2017. All patients were pathologically diagnosed with pHL by surgical biopsy or percutaneous lymph node biopsy. We excluded patients with (1) only a short-term follow-up period, or (2) mantle-field RT. Patients were included if they received CMT with chemotherapy followed by RT with IFRT or INRT. Approval by the Institutional Review Board of Asan Medical Center was obtained for this study (IRB No. 2018-0774).

Patients were physically examined and underwent chest X-ray and computed tomography (CT) of the neck, chest, abdomen, and pelvis. Patients who were diagnosed after 2006 could also undergo additional positron emission tomography combined with computed tomography (PET-CT). Bone marrow aspiration was performed for the exclusion of bone marrow involvement.

Patients were staged according to the Cotswold modification of Ann Arbor Staging (AAS). Bulky disease was defined as the longest diameter of a lymph node (LN) $>6 \mathrm{~cm}$ or a mediastinal mass with more than one-third of the maximal thoracic diameter. B symptoms were defined as unexplained fever $>38^{\circ} \mathrm{C}$, unexplained weight loss $>10 \%$ within the last 6 months, and/or night sweats.

We classified the risk of the patients based on their initial stage, the presence of bulky disease, and the presence of B symptoms. The low-risk group was defined as AAS I-II without adverse factors (B symptoms or bulky disease). The intermediate-risk group was defined as AAS I-II with adverse factors or AAS III without adverse factors. The high-risk group was AAS III-IV with adverse factors. The details of the patient classification and risk-stratification are described in Fig. 1.
All patients received chemotherapy before RT. The chemotherapy regimens varied by their risk classification. The majority of the patients received chemotherapy based on the Children's Oncology Group (COG) protocols with risk-adapted therapy [8-10,12]. Patients diagnosed before the development of the $\mathrm{COG}$ protocols received ABVD (doxorubicin, bleomycin, vinblastine, and dacarbazine) or MOPP (nitrogen mustard, vincristine, prednisone, and procarbazine) based regimens, which originated from studies of adult Hodgkin lymphoma. We assessed the disease response after completion of the chemotherapy as a 1st response or after the number of cycles that each protocol specified.

Patients received RT within 1 month after the completion of chemotherapy. We delineated the target volumes based on the guidelines for IFRT and INRT $[13,14]$. Gross target volume (GTV) was defined as visibly involved LNs based on the pre- and post-chemotherapy PET-CT. For IFRT, clinical target volume (CTV) covered the main involved lymph node region in the pre-treatment CT or PET-CT. For INRT, we considered the initial volume of the involved LNs or residual involved LNs on post-treatment PET-CT to contour the CTV. We contoured the planning target volume (PTV) by extending 7-10 $\mathrm{mm}$ from the CTV, considering organ movements and set-up variations. We delivered 19.8-36 Gy to the PTV with a fraction size of 1.5-2.0 Gy. In particular, patients with a complete remission (CR) at the 1st response received $\mathrm{RT}$ with a dose less than 30.6 Gy. After the results from the COG protocols for pediatrics and young adults were published, our institution further decreased the dose for CR patients to $25.2 \mathrm{~Gy}[8,10]$. Patients who did not achieve a CR at the 1st response received boost RT to the residual involved LN. Overall, we mostly delivered a total RT dose under $40 \mathrm{~Gy}$.

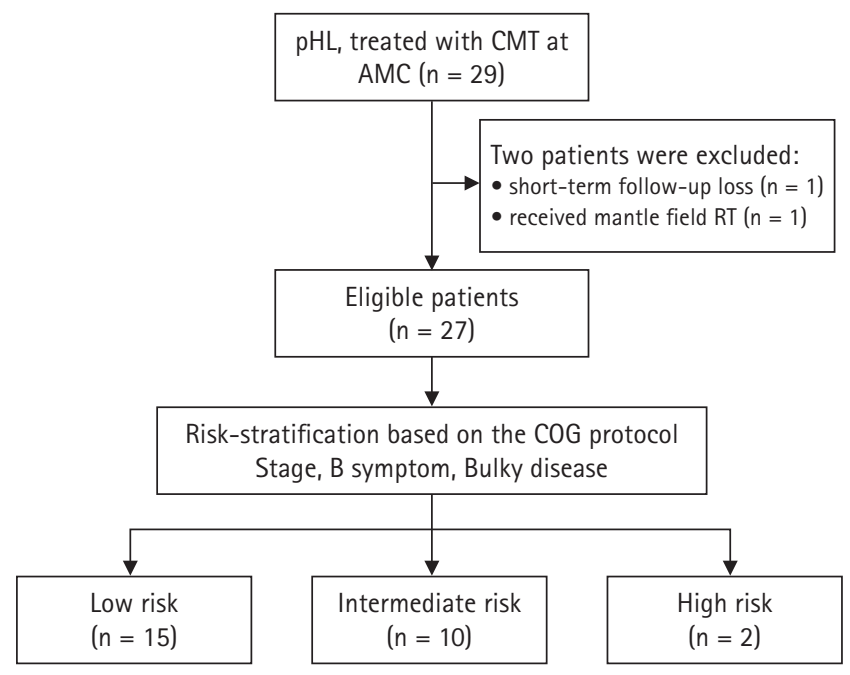

Fig. 1. Flow chart with selection criteria and risk-stratification. $\mathrm{pHL}$, pediatric Hodgkin lymphoma; CMT, combined modality treatment; AMC, Asan Medical Center; COG, Children's Oncology Group. 
After completion of CMT, we performed a physical examination; neck, chest, and abdominopelvic CT and/or PET-CT as the final response assessment. Non-complete responders (non-CR) subsequently received additional treatment. Regular follow-ups with whole-body CT or MRI were performed every 6 months until 2 years after the completion of treatment, and every 1 year after that.

A CR was defined as a more than $70 \%$ decrease of the sum of the products of the perpendicular diameters of measurable lesions (SPPD) for the patients with CT only [8]. For the patients with PETCT, a CR was defined as Deauville Criteria 1 to 3 [15]. A partial response (PR) was defined as a decrease of SPPD between 50\%-70\% for CT only, and Deauville Criteria 4 without any new lesions.

Acute and late toxicities were evaluated based on the pre- and post-treatment general blood chemistry, thyroid function test, post-treatment imaging, pulmonary function tests, echocardiograms, and systemic review. We evaluated acute toxicities during treatment and the 1 month after completion of CMT. Acute toxicities were divided into chemotherapy-induced and RT-induced toxicities. Thyroid function and pulmonary function tests were conducted for patients who received RT to these organs or by the clinician's decision. Patients with a high cumulative anthracycline dose underwent an echocardiogram at least once after the CMT. We evaluated the severity of the toxicities with the Common Terminology Criteria for Adverse Events version v4.03 [16].

We used the Kaplan-Meier method for survival analysis with SPSS software for Windows (version 22.0; IBM, Armonk, NY, USA). OS was defined as the time from the start of the combined therapy to any cause of death. EFS was defined as the time from the start of the combined therapy to the progression or relapse of the disease, or the occurrence of a secondary malignancy.

\section{Results}

\section{Patient characteristics}

A total of 27 pediatric patients who received CMT for $\mathrm{pHL}$ were analyzed (Table 1). The median age at diagnosis was 14 years (range, 4 to 19 years). The male to female ratio was 1.7:1. The majority of patients had only supradiaphragmatic involvement $(n=$ 22; 81.5\%). Cervical lymphadenopathy was the most frequently involved region ( $n=25 ; 92.6 \%$ ), followed by mediastinal lymphadenopathy ( $\mathrm{n}=16 ; 59.3 \%)$.

Histopathologic subtypes were classified as follows: nodular sclerosis (17), mixed cellularity (6), lymphocyte predominant (3), and not specified (1). B symptoms were observed in only 4 patients, and 3 of them had night sweats while 1 had unexplained fever. Seven patients had bulky disease (25.9\%). A total of 6 patients had extranodal involvement: spleen (4) and lung (2).

We stratified patients based on the COG risk-stratification protocol. Fourteen patients were low-risk, 11 patients were intermediate, and 2 patients were high-risk. Patients were treated based on the risk-adapted chemotherapy regimens.

\section{Treatment and response}

We assessed the response to chemotherapy by the initial staging method, CT, or PET-CT. PET-CT was applied after 2006, and only 8 patients (29.6\%) underwent PET-CT. Thirteen patients were considered to have a CR (48.1\%) at the 1st response. Only one patient had progressive disease. Within the patients who underwent PETCT, 4 patients were considered to have a CR (50\%) at the 1st response.

After the completion of chemotherapy, all 27 patients received IFRT or INRT. Among these patients, 14, 12, and 1 received 2D, 3D, and intensity-modulated radiotherapy (IMRT), respectively. A comparison of the $2 \mathrm{D} / 3 \mathrm{D}$-conformal radiotherapy (CRT) and the IMRT is presented in Fig. 2. The median RT dose was 25.2 Gy (range, 19.8 to

Table 1. Patients' characteristics $(n=27)$

\begin{tabular}{|c|c|}
\hline Characteristic & Value \\
\hline Age (yr) & $14(4-19)$ \\
\hline \multicolumn{2}{|l|}{ Sex } \\
\hline Male & $17(63.0)$ \\
\hline Female & $10(37.0)$ \\
\hline \multicolumn{2}{|l|}{ Ann Arbor Staging } \\
\hline I & $5(18.5)$ \\
\hline II & $17(63.0)$ \\
\hline III & 5 (18.5) \\
\hline \multicolumn{2}{|l|}{ Histologic type } \\
\hline Nodular sclerosis & $17(63.0)$ \\
\hline Mixed cellularity & $6(22.2)$ \\
\hline Lymphocyte predominant & $3(11.1)$ \\
\hline Not otherwise specified & $1(3.7)$ \\
\hline \multicolumn{2}{|l|}{ Lymphadenopathy } \\
\hline Cervical & $25(92.6)$ \\
\hline Mediastinal & $16(59.3)$ \\
\hline Axilla & $6(22.2)$ \\
\hline \multicolumn{2}{|l|}{ B symptoms } \\
\hline Yes & $4(14.8)$ \\
\hline No & $23(85.2)$ \\
\hline \multicolumn{2}{|l|}{ Bulky disease } \\
\hline Yes & $7(25.9)$ \\
\hline No & $20(74.1)$ \\
\hline \multicolumn{2}{|l|}{ Extranodal involvement } \\
\hline Yes & $6(22.2)$ \\
\hline No & $21(77.8)$ \\
\hline
\end{tabular}

Values are presented as median (range) or number (\%). 

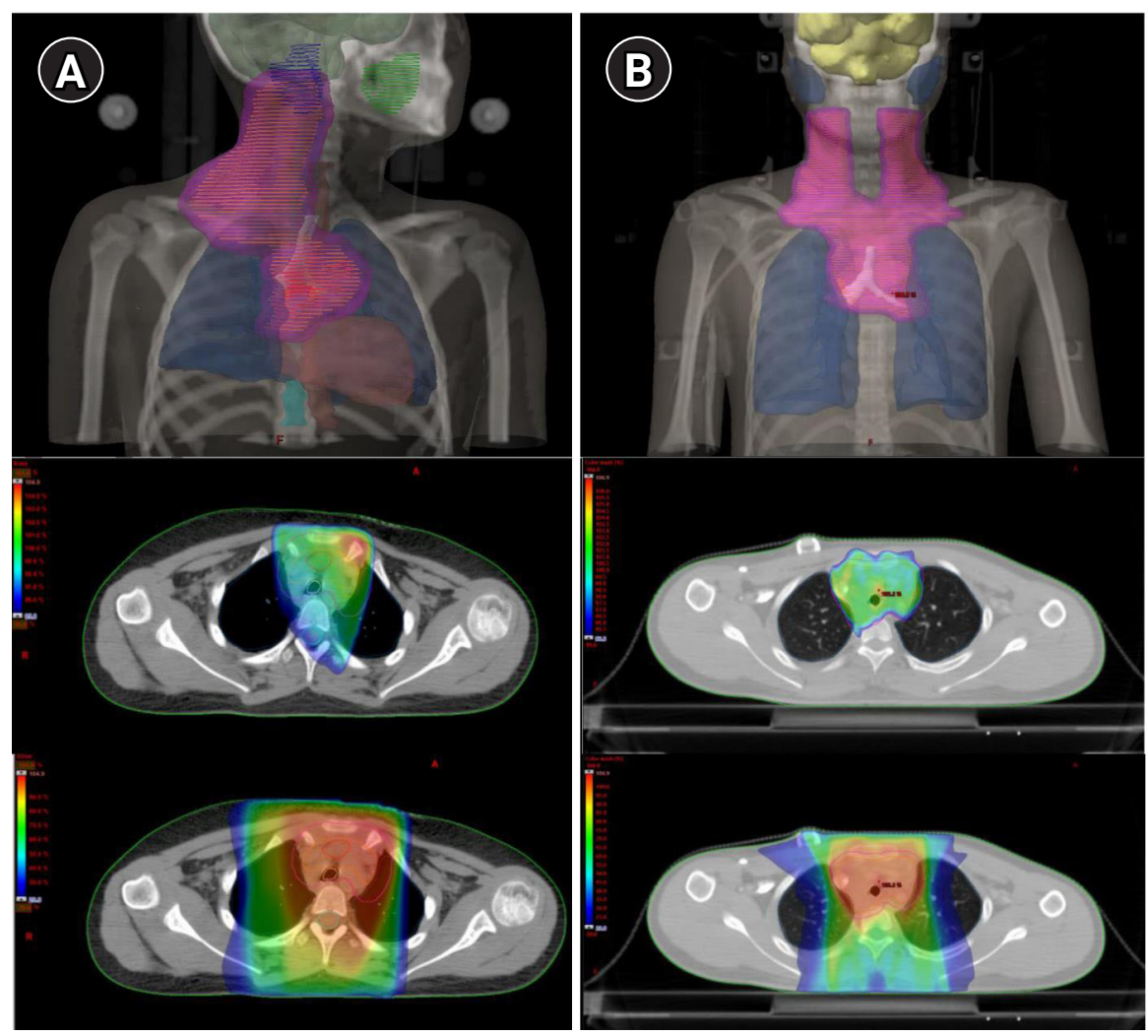

Fig. 2. Comparison of target volume contouring and dose distribution of (A) 3D-CRT and (B) IMRT. (A) 3D-CRT with 4 beams (AP/PA, LAO, RAO). (B) Nine static fields were used in this patient. 3D-CRT, three-dimensional conformal radiotherapy; IMRT, intensity-modulated radiotherapy; AP/ $\mathrm{PA}$, anterior-posterior/posterior-anterior; LAO, left anterior oblique; RAO, right anterior oblique.

36 Gy). The median fraction size was $1.8 \mathrm{~Gy} /$ fraction (range, 1.5 to $2.0 \mathrm{~Gy} /$ fraction). Based on the response to the chemotherapy, the prescribed RT dose was different. For the $18 \mathrm{CR}$ patients, the median RT dose was 25.2 Gy (range, 19.8 to $41.2 \mathrm{~Gy}$ ). Nine non-CR patients received additional $\mathrm{RT}$ to the residual LNs. The median RT dose of the non-CR groups was 35.6 Gy (range, 25.2 to $40.6 \mathrm{~Gy}$ ). The total RT dose of the non-CR group was significantly higher ( $p$ $<0.001)$.

A CR was achieved in 23 patients after CMT (85.2\%). Five patients achieved a CR out of 6 patients with subsequent PET-CT. Five patients received additional chemotherapy: 3 cases with a PR and 1 case with continuous progression. One patient achieved a CR based on PET-CT, but was suspicious for residual disease on enhanced $\mathrm{CT}$, so additional chemotherapy was prescribed. Except for the patient with progressive disease, 3 patients with a PR after CMT achieved a CR after additional chemotherapy. A summary of the CMT of each patient is presented in Table 2.

\section{Oncologic outcomes}

The median follow-up period of the patient group was 125 months (range, 9 to 337 months). The 10-year EFS and OS were $88.9 \%$ and
96.3\%, respectively (Fig. 3).

Only 2 patients had relapsed or progressive disease after CMT. The patient with progressive non-responsive disease mainly progressed in the mediastinum with tracheal obstruction, so this patient first received boost RT of 20 Gy to the mediastinal bulky mass and subsequently received IFRT that targeted the residual LNs. This patient subsequently received ESHAP (etoposide, solumedrol, ara-C, cisplatin) and two cycles of DICE (dexamethasone, ifosfamide, cisplatin, etoposide) but died of disease progression.

The patient with relapsed mediastinal disease presented with extensive LN involvement throughout the neck, upper and lower mediastinum, and spleen involvement. This patient received $30 \mathrm{~Gy}$ of INRT targeting the neck and upper mediastinum. A relapse occurred out-of-field in the mediastinum slightly above the diaphragm. This patient subsequently received high-dose slow early response DECA (dexamethasone, etoposide, cisplatin and cytarabine) chemotherapy supported by peripheral blood stem cell transplantation and regained a CR status.

\section{Toxicities and secondary malignant neoplasms}

Acute toxicities were mainly hematologic toxicity due to the che- 
Table 2. Summary of combined modality treatment of pediatric Hodgkin lymphoma patients

\begin{tabular}{|c|c|c|c|c|c|c|c|c|}
\hline Patient\# & Diagnosis year & Risk group & Chemotherapy (cycle) & RT dose (Gy) & RT method & Response & Event & Status at follow-up \\
\hline 1 & 1990 & Low & ABVD/MOPP (6) & 19.8 & $2 \mathrm{D}$ & $\mathrm{CR}$ & - & CR at $337 \mathrm{mo}$ \\
\hline 2 & 1991 & Low & MOPP (8) & 19.8 & $2 \mathrm{D}$ & $\mathrm{CR}$ & - & $\mathrm{CR}$ at $330 \mathrm{mo}$ \\
\hline 3 & 1993 & Low & MOPP+COPP (8) & 30.6 & $2 \mathrm{D}$ & $\mathrm{CR}$ & - & $\mathrm{CR}$ at $65 \mathrm{mo}$ \\
\hline 4 & 1995 & Low & COPP (4) & 23.4 & $2 \mathrm{D}$ & $\mathrm{CR}$ & Thyroid cancer, $7.2 \mathrm{yr}$ & $\mathrm{CR}$ at $280 \mathrm{mo}$ \\
\hline 5 & 1998 & Low & ABVD (6) & 28 & $2 \mathrm{D}$ & $\mathrm{CR}$ & - & $\mathrm{CR}$ at $96 \mathrm{mo}$ \\
\hline 6 & 1998 & Low & ABV/COPP (4) & 25.2 & $2 \mathrm{D}$ & $\mathrm{CR}$ & - & $\mathrm{CR}$ at $55 \mathrm{mo}$ \\
\hline 7 & 2000 & Low & DBVE (2) & 25.2 & $2 \mathrm{D}$ & CR & - & $\mathrm{CR}$ at $88 \mathrm{mo}$ \\
\hline 8 & 2000 & Low & ABV/COPP (4) & 35.2 & $2 \mathrm{D}$ & $\mathrm{CR}$ & - & CR at $207 \mathrm{mo}$ \\
\hline 9 & 2002 & Low & DBVE (6) & 25.2 & $3 D$ & $\mathrm{CR}$ & - & CR at $218 \mathrm{mo}$ \\
\hline 10 & 2002 & Low & ABVD (6) & 25.5 & $2 \mathrm{D}$ & $\mathrm{CR}$ & - & CR at $136 \mathrm{mo}$ \\
\hline 11 & 2003 & Low & DBVE (4) & 25.2 & $2 \mathrm{D}$ & $\mathrm{CR}$ & - & $\mathrm{CR}$ at $127 \mathrm{mo}$ \\
\hline 12 & 2004 & Low & DBVE (4) & 36 & $2 \mathrm{D}$ & $\mathrm{CR}$ & - & CR at $156 \mathrm{mo}$ \\
\hline 13 & 2005 & Low & DBVE (4) & 36 & $3 D$ & CR & - & $\mathrm{CR}$ at $123 \mathrm{mo}$ \\
\hline 14 & 2006 & Low & DBVE (4) & 40.6 & $3 D$ & PR & - & $\mathrm{CR}$ at $113 \mathrm{mo}$ \\
\hline 15 & 2007 & Low & AVPC (4) & 41.2 & $3 D$ & CR & - & CR at $157 \mathrm{mo}$ \\
\hline 16 & 1997 & Intermediate & MOPP (4) & 30.6 & $2 \mathrm{D}$ & $\mathrm{CR}$ & - & CR at $257 \mathrm{mo}$ \\
\hline 17 & 1998 & Intermediate & ABV/EDAP (10) & 39.6 & $2 \mathrm{D}$ & $\mathrm{CR}$ & - & $\mathrm{CR}$ at $211 \mathrm{mo}$ \\
\hline 18 & 2004 & Intermediate & ABVD (6) & 25.2 & $2 \mathrm{D}$ & $\mathrm{CR}$ & - & $\mathrm{CR}$ at $151 \mathrm{mo}$ \\
\hline 19 & 2006 & Intermediate & ABVE-PC (6) & 40 & $3 D$ & PR & - & $\mathrm{CR}$ at $125 \mathrm{mo}$ \\
\hline 20 & 2006 & Intermediate & ABVD (4) & 30.6 & $3 D$ & $\mathrm{CR}$ & - & $\mathrm{CR}$ at $58 \mathrm{mo}$ \\
\hline 21 & 2009 & Intermediate & ABVE-PC (4) & 30 & $3 D$ & PR & - & $\mathrm{CR}$ at $89 \mathrm{mo}$ \\
\hline 22 & 2011 & Intermediate & ABVE-PC (4) & 30 & $3 \mathrm{D}$ & $\mathrm{CR}$ & - & $\mathrm{CR}$ at $105 \mathrm{mo}$ \\
\hline 23 & 2012 & Intermediate & ABVD (3) & 36.2 & $3 D$ & PD & $\begin{array}{l}\text { Died of disease } \\
\text { progression }\end{array}$ & Died at 9 mo \\
\hline 24 & 2016 & Intermediate & ABVE-PC (6) & 25.2 & $3 \mathrm{D}$ & $\mathrm{CR}$ & - & $\mathrm{CR}$ at $37 \mathrm{mo}$ \\
\hline 25 & 2017 & Intermediate & ABVE-PC (6) & 30 & IMRT & $\mathrm{CR}$ & Mediastinal relapse & $\mathrm{CR}$ at $34 \mathrm{mo}$ \\
\hline 26 & 2005 & High & DBVE (4) & 36 & $3 \mathrm{D}$ & $\mathrm{CR}$ & - & $\mathrm{CR}$ at $151 \mathrm{mo}$ \\
\hline 27 & 2015 & High & ABVE-PC (5) & 21 & $3 \mathrm{D}$ & $\mathrm{CR}$ & - & $\mathrm{CR}$ at $58 \mathrm{mo}$ \\
\hline
\end{tabular}

$R T$, radiotherapy; $C R$, complete response; $P R$, partial response; $P D$, progressive disease; $A B V D$, doxorubicin, bleomycin, vinblastine, and dacarbazine; MOPP, nitrogen mustard, vincristine, prednisone, and procarbazine; ABV/COPP, doxorubicin, bleomycin, and vinblastine/cyclophosphamide, vincristine, procarbazine, and prednisone; EDAP, etoposide, dexamethasone, cytarabine, and cisplatin; DBVE, doxorubicin, bleomycin, vincristine, and etoposide; ABVE-PC, doxorubicin, bleomycin, vincristine, etoposide, prednisone, and cyclophosphamide.
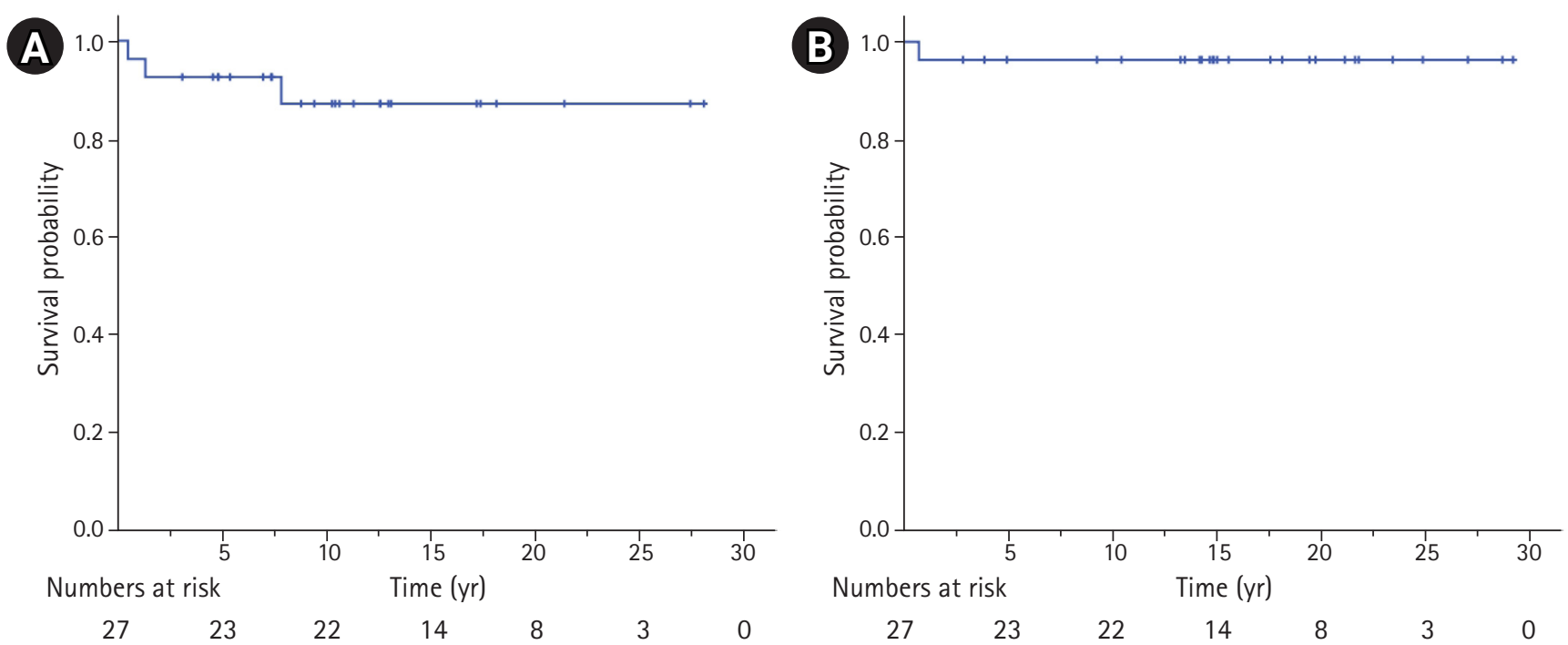

Fig. 3. Kaplan-Meier curves for (A) event-free survival and (B) overall survival in patients with pediatric Hodgkin lymphoma. 
motherapy. Severe neutropenia (Grade $\geq 3$ ) occurred in 18 patients. We observed 8 patients with febrile neutropenia during chemotherapy that required admission and treatment with intravenous antibiotics. Severe thrombocytopenia and anemia were recorded in 6 and 2 patients, respectively.

There was no severe non-hematologic RT-induced toxicity observed. Grade II esophagitis and cough were observed in only one patient each. High dose RT did not show a higher incidence of RT-induced toxicity. We did not observe any long-term sequelae from severe acute toxicity. Details of the acute toxicities are described in Table 3.

The median follow-up period for the surviving patients was 126 months (range, 34 to 337 months). We performed echocardiograms in 10 out of 26 survivors (38.5\%) after completion of the CMT. There was no abnormal echocardiogram observed.

Of the 13 patients who underwent a pulmonary function test before the treatment, 8 patients received a follow-up pulmonary function test after completion of the treatment. Only one patient had a mild obstructive pattern, with a decreased forced volume capacity and an FEV1 of $1.68 \mathrm{~L}$ (68\% predicted) and $1.68 \mathrm{~L}$ (73\% predicted), respectively. This patient received 4 cycles of ABVE-PC (doxorubicin, bleomycin, vincristine, etoposide, prednisone, and cyclophosphamide) and $30 \mathrm{~Gy}$ of RT to the mediastinum.

Thyroid function test were conducted in 12 patients (44.4\%) among 23 patients with neck irradiation. Thyroid hormone-replacement therapy was required in 7 patients (58.3\%) who underwent thyroid testing. All patients with hormonal replacement had received $\mathrm{RT}$ to the neck. The median RT dose of the patients with thyroid-hormone replacement was $30 \mathrm{~Gy}$ (range, 19.8 to $41.2 \mathrm{~Gy}$ ), whereas that of the patients without thyroid toxicity was $30.3 \mathrm{~Gy}$ (range, 19.8 to $40.6 \mathrm{~Gy}$ ). Also, RT techniques did not differ between the two groups.

We observed only one secondary malignant neoplasm (SMN) during the follow-up period. One patient experienced a non-hematologic secondary malignancy, a papillary thyroid cancer that was diagnosed 7.2 years after $23.4 \mathrm{~Gy}$ of neck irradiation. There were no secondary hematologic malignancies observed.

\section{Discussion and Conclusion}

Pediatric Hodgkin lymphoma is a curable disease with an extremely low incidence in childhood and adolescence. pHL shows a bimodal age-specific distribution and it is the third most common pediatric malignancy in the United States [2]. Long-term OS and EFS of pHL exceeds $90 \%$ in western reports regardless of the treatment method [3].

The clinicopathology of $\mathrm{HL}$ is different among different ethnic groups [4]. A lower incidence is observed in Asia compared with North America and Australia. Studies conducted in India showed a male predominance, a younger age at presentation, and an increased incidence of a mixed cellularity subtype than in western reports [17]. Hwang et al. [11] reported a change in the pathologic pattern from mixed cellularity predominance to nodular sclerosis predominance. We also observed a similar pattern of $\mathrm{pHL}$ with a male (63\%) and nodular sclerosis subtype predominance. Kobayashi et al. [18] also reported a male predominance and a low incidence of 20-30 patients per year in Japan. Park et al. [1] reported that the crude incidence was 1.3 per million among those 0 to 14 years of age, and did not observe a bimodal increase during childhood and adolescence in Korea. Also, the majority of patients were over 10 years old at the time of diagnosis (crude incidence: $10-14$ years, 2.4 vs. $0-9$ years, 1.3$)$. Similarly, only 6 patients $(22 \%)$ were under

Table 3. Acute toxicity after combined modality treatment

\begin{tabular}{|c|c|c|c|c|c|c|c|c|}
\hline \multirow{2}{*}{ Variable } & \multicolumn{4}{|c|}{ Chemotherapy-induced toxicity } & \multicolumn{4}{|c|}{ Radiation-induced toxicity } \\
\hline & I & II & III & IV & I & II & III & IV \\
\hline Hematologic toxicity & - & - & - & - & - & - & - & - \\
\hline Anemia & 12 & 9 & 2 & - & - & - & - & - \\
\hline Neutropenia & 3 & 3 & 5 & 13 & - & - & - & - \\
\hline Thrombocytopenia & 9 & 1 & 4 & 2 & - & - & - & - \\
\hline Neutropenic fever & - & - & 8 & - & - & - & - & - \\
\hline Gastrointestinal toxicity & - & - & - & - & - & - & - & - \\
\hline Nausea & 1 & - & - & - & 2 & - & - & - \\
\hline Stomatitis & 3 & - & - & - & - & - & - & - \\
\hline Esophagitis & - & - & - & - & 3 & 1 & - & - \\
\hline Pulmonary toxicity & - & - & - & - & - & - & - & - \\
\hline Cough & - & - & - & - & 2 & 1 & - & - \\
\hline Dyspnea & - & - & - & - & - & - & - & - \\
\hline
\end{tabular}

Toxicities were assessed by the Common Terminology Criteria for Adverse Events (CTCAE) v4.03. 
10 years old in our study.

Even though there are discrepancies in the clinical characteristics between western and Asian countries, long-term survival and EFS are comparable (Table 4). In this study, long-term survival was comparable with other prospective studies, with 10-year OS and EFS of $96.3 \%$ and $89.3 \%$, respectively. However, patients in our study received heterogeneous treatment because of the long duration of the case collection period and the development of the riskbased chemotherapy regimen.

For the low-risk group, prospective studies have reported an excellent survival with low-dose IFRT $[9,10,19]$. There is controversy about the necessity of RT for patients with a good response after initial chemotherapy, with concerns about balancing disease control with the risk of long-term toxicities. In the CCG 5942 trial [10], patients received ABV/COPP (doxorubicin, bleomycin, and vinblastine/cyclophosphamide, vincristine, procarbazine, and prednisone) chemotherapy.

After the chemotherapy, patients with a CR were randomly assigned to 21 Gy IFRT or no RT. The 10-year EFS was significantly improved (100\% vs. $89.1 \%$ ) in response to the IFRT, whereas no benefit for survival was observed (97.1\% vs. 95.9\%). However, the POG 8625 trial [20], a randomized study of 25.5 Gy of RT or additional chemotherapy, did not report any difference in EFS or OS (EFS, $91.1 \%$ vs. 82.6\%; 0S, 96.8\% vs. 93.6\%). In the current study, there were no relapses or treatment failures in the low-risk group ( $n=15$ ), but we did observe one case of SMN that was diagnosed 7.2 years after treatment. The median RT dose was $25.5 \mathrm{~Gy}$ (range, 19.8 to $41.2 \mathrm{~Gy}$ ) in the low-risk group. Dose or field reduction of RT in low-risk groups to reduce over-treatment or long-term toxicities may be considered due to the excellent disease control observed in the current study and in other prospective study results.

Prospective trials for intermediate to high-risk patients reported EFS between $84 \%$ to $94 \%[8,10,21,22]$. In our study, 10 -year OS and EFS were $91.7 \%$ and $83.3 \%$, respectively. We observed one treatment failure and one mediastinal relapse. Both patients did not achieve a CR at the 1st response. No patients with a $\mathrm{CR}$ after chemotherapy experienced a relapse. In the CCG 5942 trial [10], the EFS in the intermediate to high-risk groups who achieved a CR after chemotherapy was not significantly improved after IFRT (intermediate, $84.0 \%$ vs. $78.0 \%$; high, $88.5 \%$ vs. $79.9 \%$ ). However, the authors did not conclude that RT was detrimental to these groups due to the small size of the groups and their need for more aggressive chemotherapy. As indicated above, RT in intermediate to highrisk patients shows mixed data, especially for patients with a $C R$ after chemotherapy. Additional studies are needed to identify the optimal RT field and dose for patients who achieve a CR after chemotherapy.

As the CMT of pHL guarantees extremely high rates of long-term survival, there have been increasing problems with serious longterm toxicities among long-term survivors, including cardiac, pulmonary, and endocrinal complications. We observed the surviving patients after a median follow-up of 126 months (range, 34 to 337 months). Because of the retrospective nature of our study, we could not obtain full imaging and chemistry work-ups for complete observations of late toxicity.

Tukenova et al. [23] reported that cumulative RT dose and anthracycline usage were associated with an increased risk of late mortality. It is known that cardiac late-toxicity is related to the cumulative anthracycline dose, and it may occur as long as 10 years after completion of therapy with a low cumulative anthracycline dose [24]. Most of our patients received anthracycline-based chemotherapy, and among these patients, we performed echocardiogram for 10 . We did not observe any late cardiovascular toxicity even with long-term follow-up.

Thyroid dysfunctions are the most common late toxicities, including hypothyroidism, hyperthyroidism, and secondary thyroid

Table 4. Treatment outcomes of prospective trials and current study

\begin{tabular}{|c|c|c|c|c|c|}
\hline Study & Risk group & Number of patients & Chemotherapy & Radiation dose (Gy) & EFS (\%) \\
\hline CCG 5942 [10] & All & 826 & 4-6 COPP/ABV & 21 & $83.5(10-y r)$ \\
\hline POG 9426 [9] & Low & 255 & 2-4 ABVE & 25.5 & $88.3(5-y r)$ \\
\hline \multirow[t]{2}{*}{ AHOD 0431 [19] } & Low & 175 & & CR: No-RT & 77.5 (4-yr) \\
\hline & & 100 & & PR/SD: 21 & 82.8 \\
\hline \multirow[t]{3}{*}{ AHOD $0031[12,22]$} & Intermediate-high & 382 & 4-6 ABVE-PC & RER, CR: No-RT & 86.7 (4-yr) \\
\hline & & 380 & & RER, CR: 21 & 87.3 \\
\hline & & 305 & & SER: 21 & 77.4 \\
\hline POG 9425 [8] & High & 216 & 3-5 ABVE-PC & 21 & $84.0(5-y r)$ \\
\hline Current study & All & 27 & Heterogeneous & $19.8-41.2$ & $88.9(10-y r)$ \\
\hline
\end{tabular}

$\mathrm{CR}$, complete response; $\mathrm{PR}$, partial response; $\mathrm{PD}$, progressive disease; $\mathrm{RT}$, radiotherapy; EFS, event-free survival; RER, rapid early response; SER, slow early response; COPP/ABV, cyclophosphamide, vincristine, procarbazine, and prednisone/ doxorubicin, bleomycin, and vinblastine; ABVD, doxorubicin, bleomycin, vinblastine, and dacarbazine; ABVE-PC, doxorubicin, bleomycin, vincristine, etoposide, prednisone, and cyclophosphamide. 
cancer. Sklar et al. [25] reported that 34\% of patients were diagnosed with at least one type of thyroid dysfunction, and hypothyroidism was the most common dysfunction. The risk for thyroid dysfunction is 4-5 times higher after neck irradiation. Hancock et al. [26] observed 50\% of patients had 20-year long-term thyroid toxicity after mantle-field RT. The RT dose to the thyroid gland is also an important factor for thyroid dysfunction. Thyroid $V_{30}$ is an independent predictor of hypothyroidism. In patients with thyroid $V_{30}>62.5 \%$, the incidence of hypothyroidism is significantly increased (high-volume 70.8\% vs. low-volume 11.5\%; $p<0.01$ ) [27]. Even though we observed a similar incidence as in previous studies with larger field RT, the actual proportion of thyroid toxicities in our group may be lower because only half of the patients underwent thyroid function test. Additional dose-sparing for the thyroid by block or conformal therapy is needed to decrease long-term thyroid toxicity further.

SMN is associated with the RT dose and the use of alkylating agents or anthracyclines $[28,29]$. Common SMNs are leukemia, sarcoma, thyroid, and breast cancers. However, the incidence of SMN does not change linearly in response to the RT dose or field. The risk of thyroid cancer is the highest at $20 \mathrm{~Gy}$, and then it decreases as the RT dose increases [30]. We observed a patient with papillary thyroid cancer who received 23.4 Gy neck irradiation, which is consistent with previous reports. We did not observe any SMNs after higher irradiation doses.

There are several limitations to our current study. First, we analyzed a small number of patients over 30 years. Due to the small number of patients and the high disease control rate, we could not evaluate the prognostic factors for $\mathrm{pHL}$. Moreover, the patients received heterogeneous chemotherapy based on risk-grouping. The current study included all risk groups and included some patients who were diagnosed before the development of specific chemotherapy regimens for children, which led to the use of diverse chemotherapy regimens. Thus, it is hard to determine the necessity of RT or to evaluate long-term toxicities in this patient group. In addition, the RT technique and protocols have evolved over the last 30 years, and thus the patients received heterogeneous RT techniques, including 2D, 3D-CRT, and IMRT. However, this study has a strength in that the same medical team used consistent decision criteria, and there were a relatively large number of cases, despite the low incidence of pHL in Korea. Thus, there was some homogeneity of treatment provided by the same medical team. The current study has clinical meaning because it only assessed survival and long-term toxicity after CMT with IFRT or INRT for pHL. Further investigation with multicenter, large-scale studies is needed to optimize the treatment with lower toxicity, but the findings of the current study strengthen the evidence base supporting CMT with a smaller RT field in Korea.

In conclusion, CMT with IFRT and INRT may lead to favorable long-term survival. Smaller field RT could be considered as an adaptive treatment option with lesser long-term toxicities as compared with high-dose, large-field RT.

\section{Conflict of Interest}

No potential conflict of interest relevant to this article was reported.

\section{References}

1. Park HJ, Moon EK, Yoon JY, et al. Incidence and survival of childhood cancer in Korea. Cancer Res Treat 2016;48:869-82.

2. Ries LA, Smith MA, Gurney JG, et al. Cancer incidence and survival among children and adolescents: United States SEER Program 1975-1999. Bethesda, MD: National Cancer Institute; 1999.

3. Olson MR, Donaldson SS. Treatment of pediatric Hodgkin lymphoma. Curr Treat Options Oncol 2008;9:81-94.

4. Cheong JW, Park SY, Roh JK, Suh CO, Hahn JS. Treatment of Hodgkin's disease: a twenty-year follow-up of patients at a center in Korea. Yonsei Med J 2006;47:455-65.

5. Henry-Amar M, Joly F. Late complications after Hodgkin's disease. Ann Oncol 1996;7 Suppl 4:115-26.

6. Bazzeh F, Rihani R, Howard S, Sultan I. Comparing adult and pediatric Hodgkin lymphoma in the Surveillance, Epidemiology and End Results Program, 1988-2005: an analysis of 21734 cases. Leuk Lymphoma 2010;51:2198-207.

7. Kelly KM, Hutchinson RJ, Sposto R, et al. Feasibility of upfront dose-intensive chemotherapy in children with advanced-stage Hodgkin's lymphoma: preliminary results from the Children's Cancer Group Study CCG-59704. Ann Oncol 2002;13 Suppl 1:107-11.

8. Schwartz $C L$, Constine LS, Villaluna $D$, et al. A risk-adapted, response-based approach using ABVE-PC for children and adolescents with intermediate- and high-risk Hodgkin lymphoma: the results of P9425. Blood 2009;114:2051-9.

9. Tebbi CK, Mendenhall NP, London WB, et al. Response-dependent and reduced treatment in lower risk Hodgkin lymphoma in children and adolescents, results of P9426: a report from the Children's Oncology Group. Pediatr Blood Cancer 2012;59:1259-65.

10. Wolden SL, Chen L, Kelly KM, et al. Long-term results of CCG 5942: a randomized comparison of chemotherapy with and without radiotherapy for children with Hodgkin's Iymphoma: a report from the Children's Oncology Group. J Clin Oncol 2012; 


\section{0:3174-80.}

11. Hwang IG, Yoo KH, Lee SH, et al. Clinicopathologic features and treatment outcomes in malignant lymphoma of pediatric and young adult patients in Korea: comparison of Korean all-ages group and Western younger age group. Clin Lymphoma Myeloma 2007;7:580-6.

12. Friedman DL, Chen L, Wolden S, et al. Dose-intensive response-based chemotherapy and radiation therapy for children and adolescents with newly diagnosed intermediate-risk Hodgkin Iymphoma: a report from the Children's Oncology Group Study AHOD0031. J Clin Oncol 2014;32:3651-8.

13. Yahalom J, Mauch P. The involved field is back: issues in delineating the radiation field in Hodgkin's disease. Ann Oncol 2002;13 Suppl 1:79-83.

14. Girinsky $T$, van der Maazen $R$, Specht $L_{\text {, }}$ et al. Involved-node radiotherapy (INRT) in patients with early Hodgkin lymphoma: concepts and guidelines. Radiother Oncol 2006;79:270-7.

15. Barrington SF, Qian W, Somer EJ, et al. Concordance between four European centres of PET reporting criteria designed for use in multicentre trials in Hodgkin lymphoma. Eur J Nucl Med Mol Imaging 2010;37:1824-33.

16. National Cancer Institute. Common Terminology Criteria for Adverse Event (CTCAE) v4.03 [Internet]. Bethesda, MD: National Cancer Institute; c2020 [cited 2020 Aug 11]. Available from: https://ctep.cancer.gov/protocolDevelopment/ electronic_applications/ctc.htm.

17. Radhakrishnan V, Dhanushkodi M, Ganesan TS, et al. Pediatric Hodgkin lymphoma treated at Cancer Institute, Chennai, India: long-term outcome. J Glob Oncol 2016;3:545-54.

18. Kobayashi R, Sunami $S$, Mitsui $T$, et al. Treatment of pediatric lymphoma in Japan: current status and plans for the future. Pediatr Int 2015;57:523-34.

19. Keller FG, Castellino SM, Chen L, et al. Results of the AHOD0431 trial of response adapted therapy and a salvage strategy for limited stage, classical Hodgkin lymphoma: a report from the Children's Oncology Group. Cancer 2018;124:3210-9.

20. Kung FH, Schwartz CL, Ferree CR, et al. POG 8625: a randomized trial comparing chemotherapy with chemoradiotherapy for chil- dren and adolescents with Stages I, IIA, IIIA1 Hodgkin Disease: a report from the Children's Oncology Group. J Pediatr Hematol Oncol 2006;28:362-8.

21. Kelly KM, Sposto R, Hutchinson R, et al. BEACOPP chemotherapy is a highly effective regimen in children and adolescents with high-risk Hodgkin lymphoma: a report from the Children's Oncology Group. Blood 2011;117:2596-603.

22. Charpentier AM, Friedman DL, Wolden S, et al. Predictive factor analysis of response-adapted radiation therapy for chemotherapy-sensitive pediatric Hodgkin lymphoma: analysis of the Children's Oncology Group AHOD 0031 Trial. Int J Radiat Oncol Biol Phys 2016:96:943-50.

23. Tukenova M, Diallo I, Hawkins M, et al. Long-term mortality from second malignant neoplasms in 5-year survivors of solid childhood tumors: temporal pattern of risk according to type of treatment. Cancer Epidemiol Biomarkers Prev 2010;19:707-15.

24. Lipshultz SE, Lipsitz SR, Sallan SE, et al. Chronic progressive cardiac dysfunction years after doxorubicin therapy for childhood acute lymphoblastic leukemia. J Clin Oncol. 2005;23:2629-36.

25. Sklar C, Whitton J, Mertens A, et al. Abnormalities of the thyroid in survivors of Hodgkin's disease: data from the Childhood Cancer Survivor Study. J Clin Endocrinol Metab 2000;85:3227-32.

26. Hancock SL, Cox RS, McDougall IR. Thyroid diseases after treatment of Hodgkin's disease. N Engl J Med 1991;325:599-605.

27. Cella $L$, Conson $M$, Caterino $M$, et al. Thyroid V30 predicts radiation-induced hypothyroidism in patients treated with sequential chemo-radiotherapy for Hodgkin's lymphoma. Int J Radiat Oncol Biol Phys 2012;82:1802-8.

28. Franklin J, Pluetschow $A_{1}$ Paus $M_{1}$, et al. Second malignancy risk associated with treatment of Hodgkin's lymphoma: meta-analysis of the randomised trials. Ann Oncol 2006;17:1749-60.

29. O'Brien MM, Donaldson SS, Balise RR, Whittemore AS, Link MP. Second malignant neoplasms in survivors of pediatric Hodgkin's lymphoma treated with low-dose radiation and chemotherapy. J Clin Oncol 2010;28:1232-9.

30. Ronckers $\mathrm{CM}$, Sigurdson $\mathrm{A}$, Stovall $M$, et al. Thyroid cancer in childhood cancer survivors: a detailed evaluation of radiation dose response and its modifiers. Radiat Res 2006;166:618-28. 